Supporting Information for

\title{
Improving the Oxygen Reduction Reaction Activity of FeN4-Graphene via Tuning Electronic Characteristics
}

\section{Lei $\mathrm{Wu}^{1}$, Xinrui Cao ${ }^{* 1,2}$, Wei $\mathrm{Hu}^{3}$, Yongfei $\mathrm{Ji}^{4}$, Zi-Zhong Zhu ${ }^{1}$ and Xiao-Fei Li ${ }^{* 5}$}

${ }^{1}$ Collaborative Innovation Center for Optoelectronic Semiconductors and Efficient Devices, Department of Physics, Jiujiang Research Institute, Xiamen University, Xiamen 361005, China

${ }^{2}$ Fujian Provincial Key Laboratory of Theoretical and Computational Chemistry, Xiamen University, Xiamen 361005, China

${ }^{3}$ Shandong Provincial Key Laboratory of Molecular Engineering, School of Chemistry and Pharmaceutical Engineering, Qilu University of Technology, Jinan, Shandong 250353, P. R. China

${ }^{4}$ School of Chemistry and Chemical Engineering, Guangzhou University, 230 Wai Huan Xi Road, Guangzhou Higher Education Mega Center, Guangzhou, 510006, P. R. China

${ }^{5}$ School of Optoelectronic Science and Engineering, University of Electronic Science and Technology of China, Chengdu, Sichuan 610054, China

*E-mail: xinruicao@xmu.edu.cn and xf.li@uestc.edu.cn

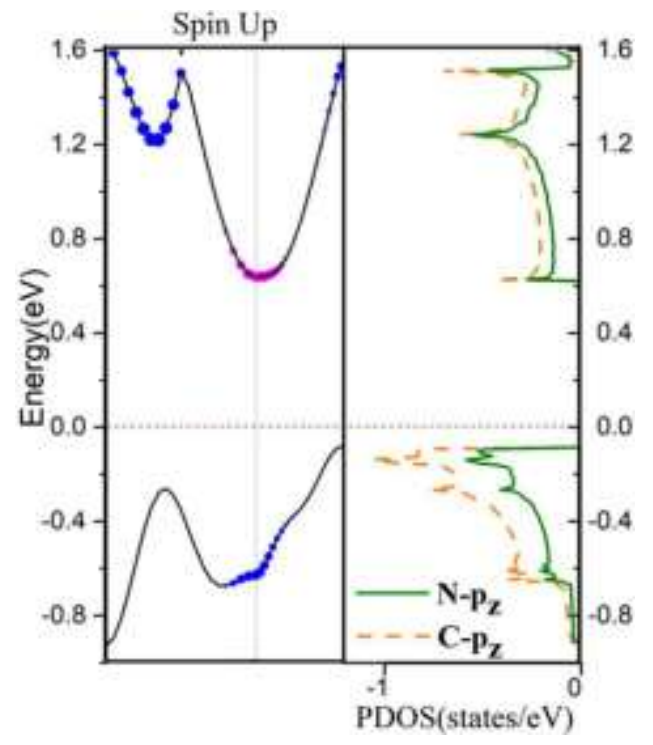

Figure S1. Band structures and Partial DOS of $\mathrm{FeN}_{4}$ embedded graphene unite cell for the spin up channel of $3 \times 3$ system. The Fermi level is set to zero. The red, blue, magenta, olive, and orange points indicate the electronic states contributed from $d_{x y}, d_{y z}, d_{x z}, d_{z}^{2}, d_{x}^{2}-y^{2}$ of Fe atom. 

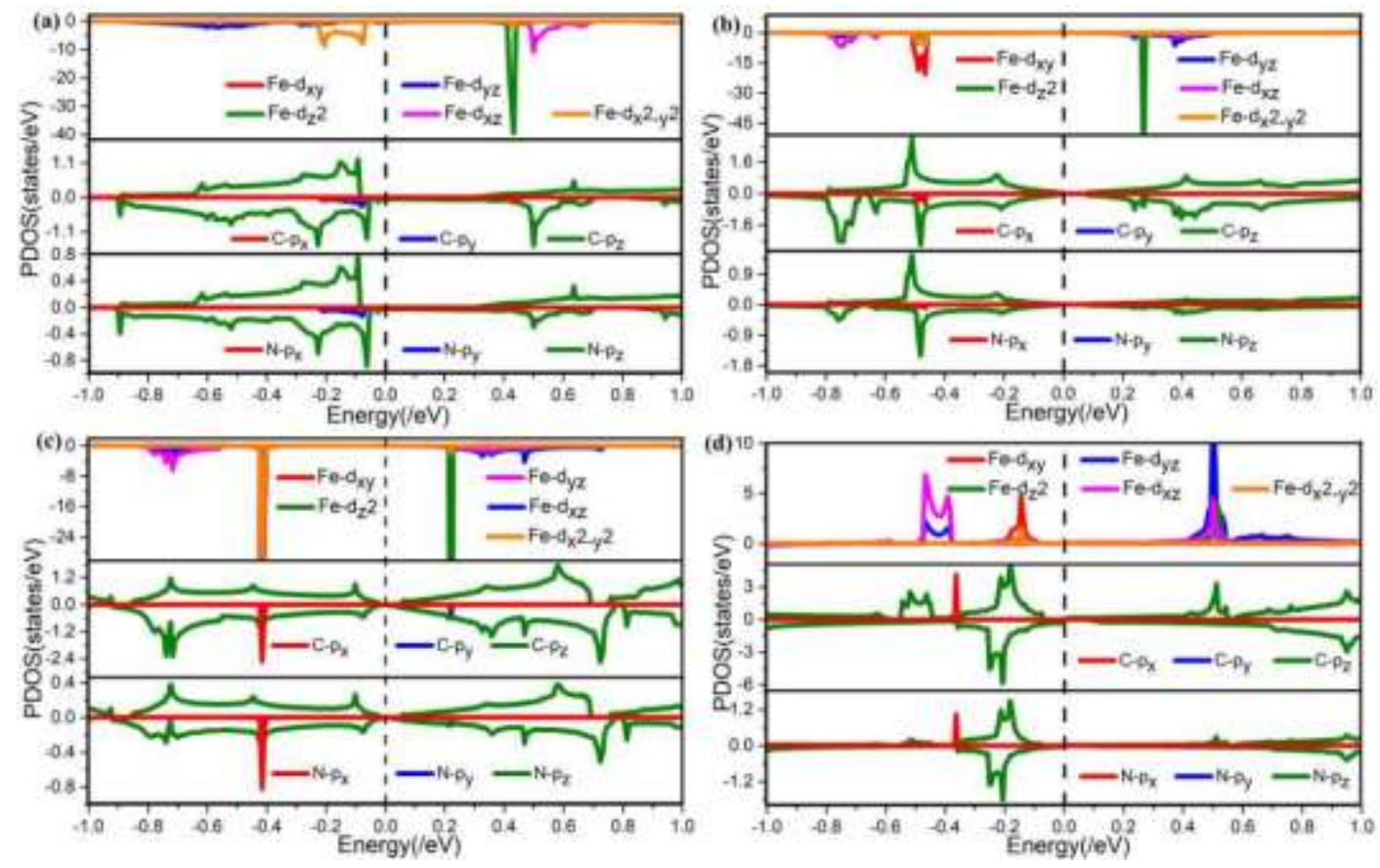

Figure S2. Partial DOS of FeN 4 embedded graphene for different systems: (a) $3 \times 3$; (b) $4 \times 4$; (c) $5 \times 5$; (d) $6 \times 6$, respectively.
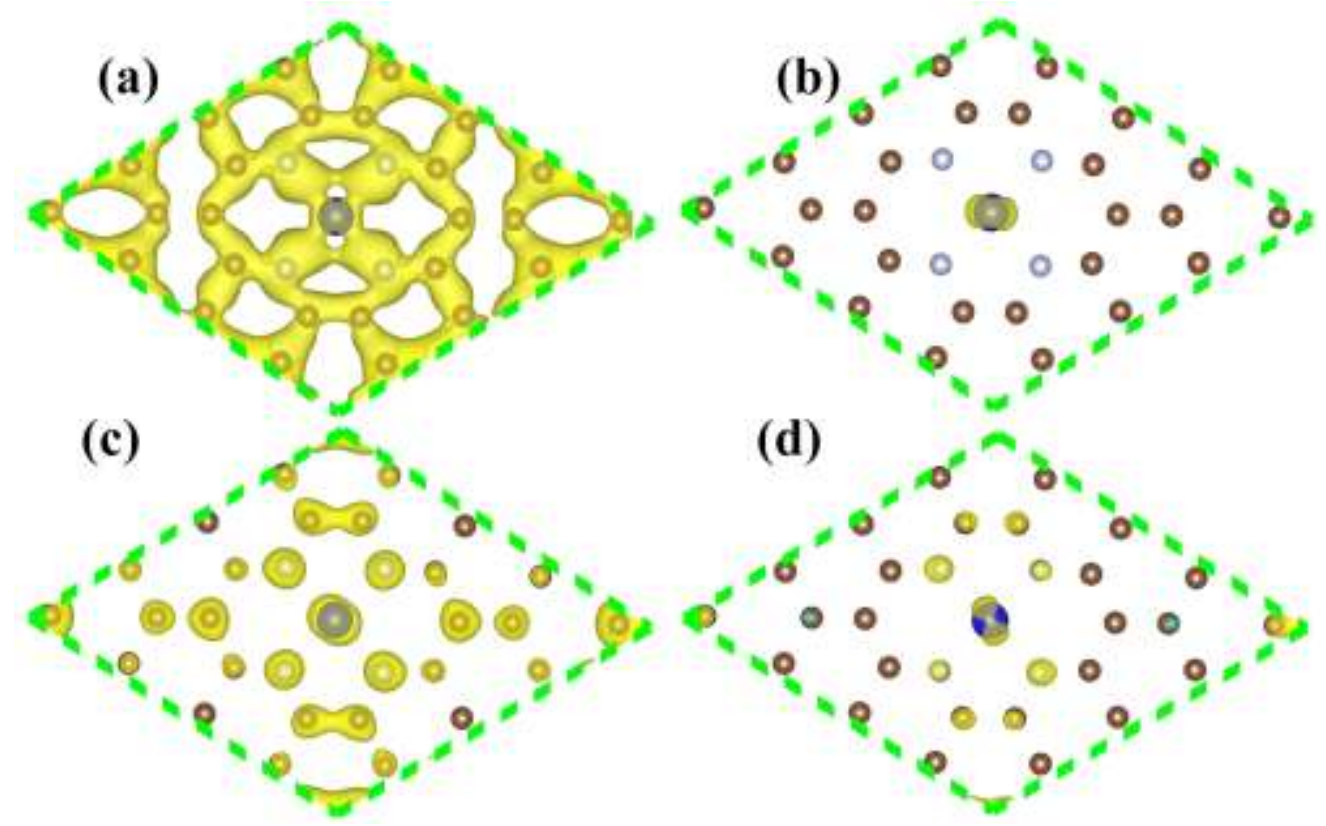

Figure S3. Calculated electron densities of the frontier bands for $4 \times 4$ system: (a) spin up and (b) spin down density of the VBM, (c) spin up and (d) spin down density of the CBM. The iso-value for charge density is 0.002 electron/ $\AA^{3}$. Yellow and cyan indicate the positive and negative densities, respectively. 


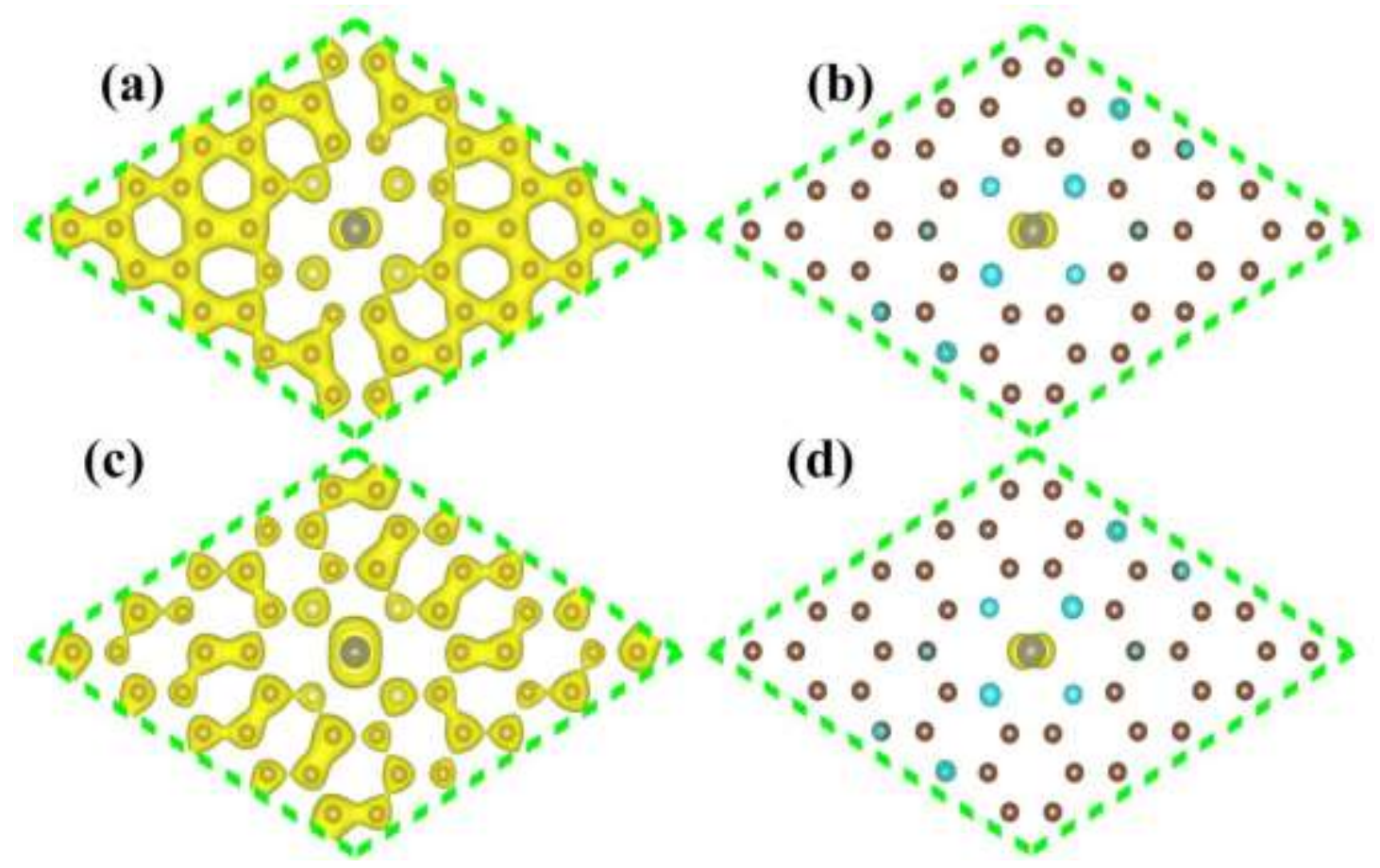

Figure S4. Calculated electron densities of the frontier bands for $5 \times 5$ system: (a) spin up and (b) spin down density of the VBM, (c) spin up and (d) spin down density of the CBM. The iso-value for charge density is 0.002 electron/ $\AA^{3}$. Yellow and cyan indicate the positive and negative densities, respectively. 
Table S1. Predicted Relative Energies $(\Delta \mathrm{E})$, Bond Lengths (d), Magnetic Moment $\left(\mu_{\mathrm{B}}\right)$, and Multiplicity of $\mathrm{O}_{2}$ Adsorbed on the $\mathrm{FeN}_{4}$ Center.

\begin{tabular}{|c|c|c|c|c|c|c|}
\hline Systems & Geometry & $d_{\mathrm{Fe}-\mathrm{O}}(\AA)$ & $d_{\mathrm{O}-\mathrm{O}}(\AA)$ & $\Delta \mathrm{E}(\mathrm{eV})$ & $\mathrm{m}\left(\mu_{\mathrm{B}}\right)$ & multiplicity \\
\hline \multirow{4}{*}{$3 \times 3$} & side-on & 1.90 & 1.35 & 0.38 & 0 & 1 \\
\hline & end-on & 1.75 & 1.29 & 0.00 & 0 & 1 \\
\hline & end-on & 1.82 & 1.30 & 0.24 & 2 & 3 \\
\hline & end-on & 2.22 & 1.27 & 0.72 & 4 & 5 \\
\hline \multirow{4}{*}{$4 \times 4$} & side-on & 1.89 & 1.37 & 0.44 & 0 & 1 \\
\hline & end-on & 1.75 & 1.29 & 0.00 & 0 & 1 \\
\hline & end-on & 1.80 & 1.30 & 0.30 & 2 & 3 \\
\hline & end-on & 2.20 & 1.27 & 0.70 & 4 & 5 \\
\hline \multirow{4}{*}{$5 \times 5$} & side-on & 1.89 & 1.37 & 0.37 & 0 & 1 \\
\hline & end-on & 1.78 & 1.30 & 0.00 & 0 & 1 \\
\hline & end-on & 1.81 & 1.30 & 0.28 & 2 & 3 \\
\hline & end-on & 2.15 & 1.28 & 0.73 & 4 & 5 \\
\hline \multirow{4}{*}{$6 \times 6$} & side-on & 1.89 & 1.37 & 0.35 & 0 & 1 \\
\hline & end-on & 1.78 & 1.30 & 0.00 & 0 & 1 \\
\hline & end-on & 1.80 & 1.30 & 0.27 & 2 & 3 \\
\hline & end-on & 2.20 & 1.28 & 0.76 & 4 & 5 \\
\hline
\end{tabular}

Table S2. Charge difference for $\mathrm{FeN}_{4}, \mathrm{C}, \mathrm{O}_{2}$ between the pure and $\mathrm{O}_{2}$-adsorbed system. (The initial charges for the aforementioned moieties are chosen as their reference values).

\begin{tabular}{|c|c|c|c|}
\hline \multirow{2}{*}{ System } & \multicolumn{3}{|c|}{$\Delta_{\text {chg }}(\mathrm{e})$} \\
\cline { 2 - 4 } & $\mathrm{FeN}_{4}$ & $\mathrm{C}$ & $\mathrm{O}_{2}$ \\
\hline $3 \times 3$ & 0.250 & -0.054 & 0.04 \\
\hline $4 \times 4$ & 0.193 & -0.034 & 0.001 \\
\hline $5 \times 5$ & 0.253 & -0.185 & 0.006 \\
\hline $6 \times 6$ & 0.132 & -0.044 & 0.008 \\
\hline
\end{tabular}


Table S3. Predicted Relative Energies $(\Delta \mathrm{E})$, Magnetic Moment $\left(\mu_{\mathrm{B}}\right)$, and Multiplicity for Dissociated $\mathrm{O}$ atoms.

\begin{tabular}{|c|c|c|c|c|c|}
\hline \multirow{2}{*}{ Systems } & \multicolumn{3}{|c|}{$\Delta \mathrm{E}(\mathrm{eV})$} & \multirow{2}{*}{$\mathrm{m}\left(\mu_{\mathrm{B}}\right)$} & \multirow{2}{*}{ multiplicity } \\
\hline & $\mathrm{T} 1 \mathrm{~T} 2$ & T1B1 & T1B2 & & \\
\hline \multirow{3}{*}{$3 \times 3$} & 0.65 & 0.32 & 0.56 & 0 & 1 \\
\hline & 0.35 & 0.00 & 0.24 & 2 & 3 \\
\hline & 1.05 & 0.76 & 0.92 & 4 & 5 \\
\hline \multirow{3}{*}{$4 \times 4$} & 0.60 & 0.30 & 0.35 & 0 & 1 \\
\hline & 0.30 & 0.00 & 0.02 & 2 & 3 \\
\hline & 0.85 & 0.88 & 0.81 & 4 & 5 \\
\hline \multirow{3}{*}{$5 \times 5$} & 0.61 & 0.30 & 0.39 & 0 & 1 \\
\hline & 0.32 & 0.00 & 0.06 & 2 & 3 \\
\hline & 0.81 & 0.83 & 0.82 & 4 & 5 \\
\hline \multirow{3}{*}{$6 \times 6$} & 0.64 & 0.31 & 0.41 & 0 & 1 \\
\hline & 0.35 & 0.00 & 0.09 & 2 & 3 \\
\hline & 0.83 & 0.90 & 0.81 & 4 & 5 \\
\hline
\end{tabular}


Table S4. Predicted Activation Energies $\left(\mathrm{E}_{\mathrm{a}}\right)$ for $\mathrm{O}_{2}$ Dissociation.

\begin{tabular}{|c|c|c|c|c|}
\hline $\mathrm{E}_{\mathrm{a}}(\mathrm{eV})$ & $\mathrm{T} 1 \mathrm{~T} 2$ & T1B1 & T1B2 & $\mathrm{m}\left(\mu_{\mathrm{B}}\right)$ \\
\hline \multirow{3}{*}{$3 \times 3$} & 1.18 & 1.18 & 1.18 & 0 \\
\hline & 0.84 & 0.84 & 0.84 & 2 \\
\hline & 1.00 & 1.00 & 1.00 & 1.27 \\
\hline \multirow{3}{*}{$4 \times 4$} & 1.40 & 1.40 & 1.51 & 0 \\
\hline & 0.98 & 0.98 & 0.98 & 2 \\
\hline & 1.26 & 1.26 & 1.26 & 1.61 \\
\hline \multirow{3}{*}{$5 \times 5$} & 1.33 & 2.07 & 1.32 & 0 \\
\hline & 0.92 & 1.69 & 0.92 & 2 \\
\hline & 1.18 & 1.92 & 1.19 & $1.60(1.24$ for $\mathrm{T} 1 \mathrm{~B} 1)$ \\
\hline \multirow{3}{*}{$6 \times 6$} & 1.35 & 2.26 & 1.34 & 0 \\
\hline & 0.97 & 1.70 & 0.96 & 2 \\
\hline & 1.23 & 1.97 & 1.23 & 1.81 \\
\hline
\end{tabular}

Table S5. Predicted Adsorption Energies $\left(E_{a d}\right)$ and Activation Energies $\left(E_{a}\right)$ for $\mathrm{O}_{2}$ end-on-singlet Adsorption and T1B2 Dissociation.

\begin{tabular}{|c|c|c|c|c|}
\hline \multirow{2}{*}{ Systems } & \multicolumn{2}{|c|}{$\mathrm{E}_{\mathrm{ad}}$} & \multicolumn{2}{c|}{$\mathrm{E}_{\mathrm{a}}$} \\
\cline { 2 - 5 } & $\mathrm{PBE}$ & $\mathrm{vdW}-\mathrm{DF}$ & $\mathrm{PBE}$ & $\mathrm{vdW}-\mathrm{DF}$ \\
\hline $3 \times 3$ & 0.91 & 1.32 & 1.00 & 0.93 \\
\hline $4 \times 4$ & 0.89 & 1.31 & 1.26 & 1.19 \\
\hline $5 \times 5$ & 0.96 & 1.37 & 1.19 & 1.11 \\
\hline $6 \times 6$ & 0.97 & 1.38 & 1.23 & 1.13 \\
\hline
\end{tabular}

